\title{
An Improved SMS User Interface Result Checking System
}

\author{
O. Awodele \\ Babcock University, Ilishan- \\ Remo, Ogun State, Nigeria
}

delealways@yahoo.com

\author{
A. T. Akinwale \\ University of Agriculture, \\ Abeokuta, Ogun State, Nigeria \\ atakinwale@yahoo.com
}

\author{
E. R. Adagunodo \\ Obafemi Awolowo University, \\ IIe-Ife, Osun State, Nigeria \\ eadigun@oauife.edu.ng
}

S. Idowu and M. Agbaje Babcock University, IlishanRemo,Ogun State, Nigeria

Saidowu06@hotmail.com agbajeolugbenga@gmail.com

\begin{abstract} comfort of their homes, on the road, or while at work. school's website.

Material published as part of this publication, either on-line or in print, is copyrighted by the Informing Science Institute. Permission to make digital or paper copy of part or all of these works for personal or classroom use is granted without fee provided that the copies are not made or distributed for profit or commercial advantage AND that copies 1) bear this notice in full and 2) give the full citation on the first page. It is permissible to abstract these works so long as credit is given. To copy in all other cases or to republish or to post on a server or to redistribute to lists requires specific permission and payment of a fee. Contact Publisher@InformingScience.org to request redistribution permission.
\end{abstract}

This paper reviews the use of mobile phones for delivering examination results via Short Messaging Service (SMS) in a university where student who have written examinations and are anxious to see their results need to get their grades in a convenient and accessible way, whether in the

At this time, a mobile phone is accessible to most students, and they take it almost everywhere with them. This technology can, therefore, be highly effective in bringing information to them quickly, easily, and while they are on the move. An SMS result checking system not only enables students to request their grades, the system can also deliver the grades to their phones as soon as the grades become available. This means that they can access their grades even in the remotest locations where internet service might be unavailable, preventing them from accessing the

This version is an improved version of the system presented previously (Adagunodo, Awodele, \& Idowu, 2009). The former system requires the user to submit an ID with a password, which is common to many SMS systems. The present system uses a social interaction with the password to reduce the incidence of guessing access codes occurring in the checking system.

Keywords: Short Message Services, SMS, GSM, Result Checking, Mobile Phone, ColdFusion

\section{Introduction}

Computers with the power of the internet have succeeded in aiding communication among people. The telephone system, which is the main communication system that was invented several 
years ago, has undergone a great improvement, so much that today we have fixed wireless phones, mobile phones, and the like. With the rapid development of mobile phones come several services, like the Short Messaging Service (SMS) and Multimedia Messaging Service (MMS), that are readily available and add to the usefulness of mobile phones. SMS in particular is widely used in communication and, more recently, has been leveraged to provide several services like airline ticketing, banking services, and commercial services such as share and sell (an added service provided by MTN Nigeria) where subscribers can easily share and/or sell airtime. SMS is a mobile technology that allows for sending and receiving text or even binary messages to and from a mobile phone. The relative ease of use of SMS makes it possible for a user to learn how to send SMS easily. More than 160 billion SMSs are exchanged each month in European countries (Mavrakis, 2004), and, according to Resource Shelf (2006), 48.7 billion SMS messages were sent in the second half of 2005, which is up 50\% from the six months before that. The major advantage of SMS is its cost effectiveness and availability, as most individuals own a phone.

In different parts of the world, several service providers offer mobile services that include SMS. Most times the cost attached to sending a SMS is relatively small, and most providers do not charge for receiving SMS. Sometimes, service providers give users a certain amount of free SMS per month, which allows customers send and receive unlimited number of SMS messages. It is possible to acquire a special dedicated line that uses a custom rate for messages sent to the number; it is also possible to have a number as toll free, making it free for users to send SMS to the number. All these contribute to what makes SMS a really cost effective means of disseminating information.

Till today, especially in the developing countries, there still exists the problem of checking examination results as students throng to notice boards in anxiety, although most universities, even those in the less developed countries, now make examination results available on their websites. The level of internet availability in less developed countries is still low and quite expensive. Even in the developed countries, where most homes have internet access, SMS is still a faster and cheaper means of disseminating examination results as well as other information. Pramsane and Sanjaya (2006) stated that universities can provide educational services based on SMS, such as grade release, enrollment information, university announcement, and internship opportunity. An advantage SMS offers is that students can receive valuable information on the fly without requesting it.

The result checking system not only allows student to request grades, it provides the result as soon as they as become available. This is done by pushing the result to the students (sending it to their phones) or working on a request sent from a student to produce the result (pulling). There are two methods of SMS widely used in applications; they are the PUSH \& PULL. This application can either be used to push or pull messages.

A Push SMS application is one whereby a message is sent from the application to the user. It is a one way message. In other words, it is the mobile application (in this case, the SMS result checking application) that initiates a message. An example could be a school that automatically sends examination results to the students and their parents/sponsors as soon as the grades become available. The users do not request the grades, do not take any action, and are not charged for receiving the SMS. The SMS would be delivered to them in a matter of seconds regardless of where they are, as long as their mobile phone is within their network operator's coverage.

A Pull SMS application, on the other hand, is one whereby a user sends a request and obtains a reply from the application. This is a full duplex scenario. An example is when a student requests for his/her grades for a recently concluded semester. 
SMS applications can be built as two kinds of services:

Independent Service - This involves using solely a mobile phone and the application server (the system running the SMS application). This option offers limited benefit, but it is easy and fast to setup. It does not require authorization of the service provider or connection to any third party SMS provider. The mobile phone uses a regular SIM card which has a normal phone number, and messages that originate from the phone attract the standard cost or tariff.

Dependent Service - This involves having the application server connect to the service provider's SMS Center (SMSC). It requires a constant connection to the internet as the application server does not require any physical phone/modem with a SIM card connected to it; rather it connects to a SMSC. When users send their request, it goes to the SMSC, which automatically forwards the message to the application server over the internet. This option provides added benefits, as the service provider can provide a special tariff and a dedicated line for the university. Here, the SMS application usually runs on corporate servers that are connected to the SMS network through specialized connectors and gateways connected to the SMS Center (SMSC) of mobile operators (Mavrakis, 2004). These servers are assigned short numbers instead of the traditional 10 or 11 digits mobile numbers. These numbers, also known as short codes, are usually 4 to 6 digits long and are operator specific. Also, a premium fee (a fee other than the fixed rates for SMS) can be charged on these short codes; in other words, users would pay more for sending SMS to short codes.

SMS uses the GSM special signaling channel instead of the voice channel and is, therefore, a very reliable media channel. Mavrakis (2004) identifies two types of SMS which can be classified by the origin of the message:

- Mobile Originated (MO): SMS-MO is sent from a mobile phone and could be sent either to another mobile phone (such as when a mobile subscriber sends a personal message to another subscriber) or to a computer application that will process the message.

- Mobile Terminated (MT): SMS-MT is transmitted to a mobile phone. It also could be sent by another mobile phone or generated by a computer application (Adagunodo, Awodele, \& Ajayi, 2007).

\section{Existing System Overview}

Most universities now provide examination results on their website. When results are made available on the website, students have to login by providing a username or ID and password before they can view their result. This ensures that examination results are protected from unauthorized access. Although websites are readily accessible and provide options like printing and saving, these benefits could easily be inaccessible if a user does not have access to the internet. Also, the student must visit the website to know find out if the examination results are available.

While websites seem to be a very good option in countries where the internet is readily available, its impact can be less felt and it can be quite inconvenient and expensive in countries with poor internet access. In such places, students will have to visit cyber cafes where they have to pay to check their grades.

Apart from using websites to provide students with their examination results, other means include IVRS (Interactive Voice Response System), and email.

\section{IVRS}

Interactive Voice Response System is a self guided result checking system. In this system, a student dials a number and listens to computer generated or recorded voice direction. The voice in- 
structs the student on what action (usually by pressing keys) to take to supply the needed information. The supplied information is then used to generate the student's result, which is spoken to the user.

\section{Email}

While email seems to be another intuitive means of sending results and other information to students, even without them requesting for it, it requires internet connection, and although several mobile phones allow accessing of emails on them, it is seldom used by mobile phone users (at least students). Email offers the benefit of mass distribution of information, which is also possible using SMS as Bulk SMS.

\section{Evaluation of Similar Systems}

\section{NTU eXpress SMS}

NeXS (NTU eXpress SMS) is used at the Nanyang Technological University. To use NeXS the user needs to be an undergraduate student of NTU and needs to register his/her mobile phone on the NeXS Portal. NeXS accepts numbers from three mobile providers (Singtel/Starhub/M1). Once a mobile phone is registered, the student can use the phone to send SMS and access information. However, students can only use the mobile number that was registered.

To get exam results via NeXS, users send the keyword NTU RESULT to 74000. If the exam results of the current semester are released, NeXS will respond with the reply (Ling, 2005).

\section{Strength of NeXS}

The primary strength of NeXS is that it offers a form of security by ensuring that only the registered mobile phone can request the result. NeXS also provides other SMS services like examination seating arrangements, subject timetable, library account information, and NTU staff directory search.

\section{Weakness of NeXS}

The fact that NeXS allows students to register their mobile phone number and limits access to the number is quite a disadvantage than an advantage. This is because it reduces the flexibility of the system and also poses a threat of identifying whether it is the real owner of the phone that is requesting for the result. For example, a student can take another student's phone and request for the student's result. NeXS cannot tell that the request wasn't made from the real owner of the phone. Also, if the register phone is stolen or lost, the student would have to visit the website to register a new phone. NeXS also provides examination result for the recently concluded semester.

\section{SMS Result Checking of Board of Secondary Education, Orissa}

The Board of Secondary Education in Orissa makes the HSC exam result available via SMS. The examination result is requested by sending the SMS Code: HSCR RollNo (for regular/regular Correspondence Courses) and HSCX Roll-No (for Ex-regular/Ex-regular Correspondence Courses) to 56505 (Desi, 2008).

\section{Weakness of the System}

The system allows requests from only BSNL Mobile and does not provide any form of security. Students can easily request the results of others by simply specifying their roll number. 


\section{MUET SMS Result Checking}

The checking of MUET Result via SMS is also another example of SMS result checking. This system receives SMS in the format MUET < space> IC Number, sent to 39003. The service charges 15 cents for each message sent and 30 cents for each received.

\section{Weakness of the System}

The system does not offer any form of security. A student with the knowledge of another student's IC number can request for that student's result. It is also expensive on the side of the users.

In summary, none of these systems provide any practical form of security and are, therefore, susceptible to several security issues. Also, they provide only the current examination results and this prevents users from requesting for older results.

\section{Proposed System}

The proposed system uses the short messaging service (SMS), which is dependent on the telecommunication infrastructure provided by GSM operators to provide a means of cheap and fast communication between the students and the university. Although a few SMS result checking systemss already implement the use of password, such a system was proposed by Pramsane and Sanjaya (2006). This system implements the same form of security and, further, provides access to new and old results. It also provides two options of requesting examination result. The SMS result checking system works using client-server architecture and can be either implemented as a dependent service or independent service. The system described here is deployed as a dependent service; this implies that the server (with the SMS application) has a phone with a standard SIM card connected to it.

The SMS server receives SMS messages from the users and processes the message by connecting to the database that holds the details and grades. The SMS server receives all SMS via the GSM terminal connected to the computer; it then connects to the database to authenticate the user and queries for the results via the appropriate database connector, like Open Database Connectivity (ODBC).

The former system (discussed in Adagunodo et al., 2009) uses a password system where the result checker submits an ID and a password). One problem is that a student may know the ID and password of another student; guessing of passwords and ID can also occur. The present system uses a social interaction mechanism which involves the use of the surname provided by the user and then a generated password to be used with an ID to reduce the incidence of guess occurring in the checking system.

\section{The UML Description of the Proposed System}

The proposed system is described below by the Unified Modeling Language (UML). The UML is a general purpose visual modeling language that is used to specify, visualize, construct, and document the artefacts of a system. It captures decision and understanding about the systems that must be constructed as depicted in the diagrams in Figure 1. This is applied to the element of the proposed system. 


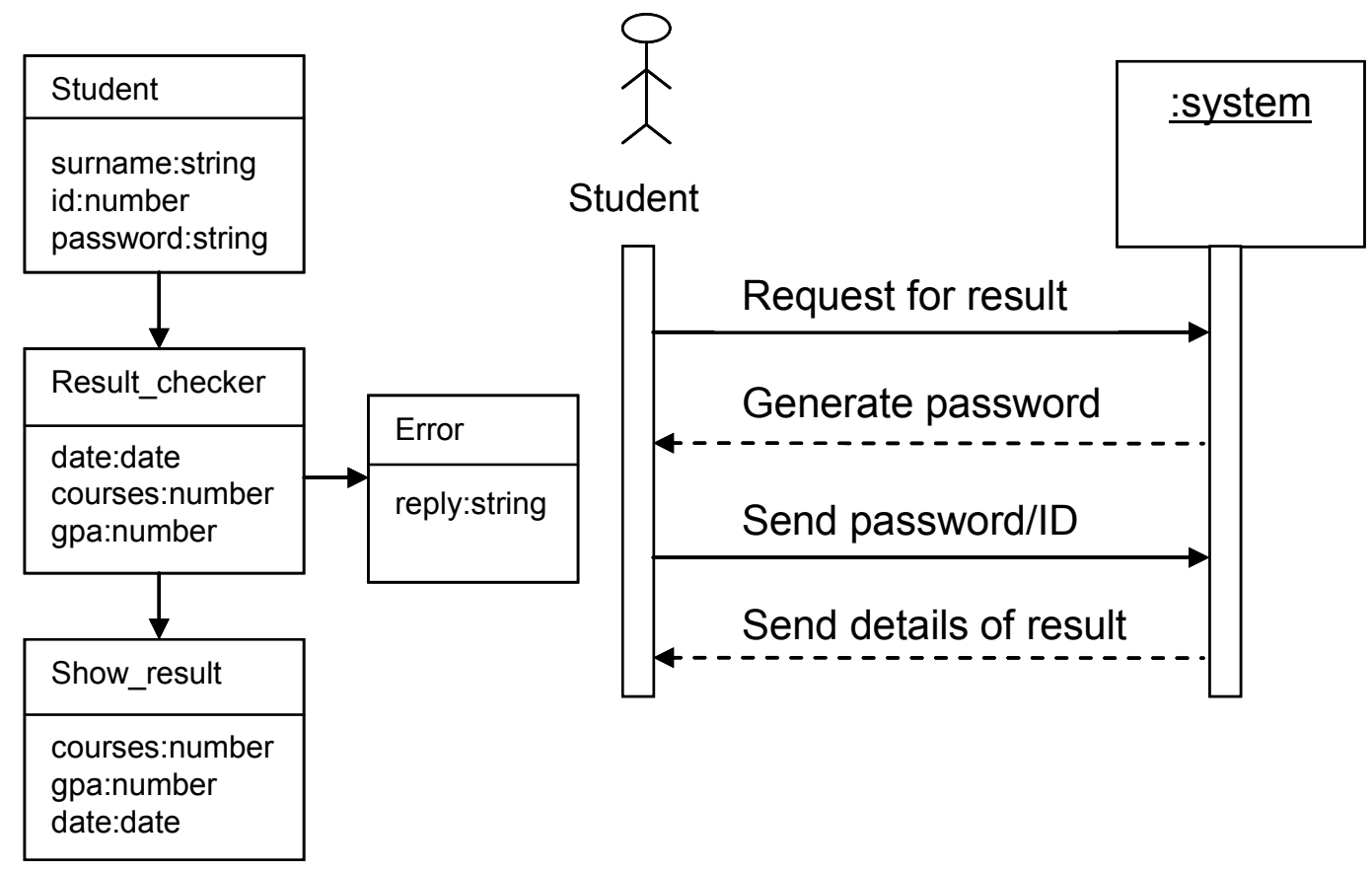

(a) Class diagram

(b) System sequence diagram

Figure 1: Class and sequence diagrams

The present system for an improved user's authentication will be a 2-tier password system that will require the student to first provide a means of personal identification for the result checking system before the system itself generates its own password. On the user's side he/she is expected to submit the surname and ID as a means of identification.

Over a period of time amongst students it has been noticed that many students do not care to know the surname of other students. Students either interact based on their nicknames or other names. Only very close friends or associates care to take note of the surname of the other. (This may not be true in other cultures; for example, in the US most students know the surnames of many others.) In a school of over three thousand students it will be difficult to know everyone surname unless you specifically print out the class list from a database.

In case of an intruder, that is, if someone seals a phone, that person can check his own results but not those of another unless he knows the surname. The trouble to him might outweigh the benefits. For the present system the student will be required to first send his/her surname and ID, which is the matriculation number of the student. The system checks if such a pair (surname, ID) exists in the result database. If such a pair exists it generates a single use password which is sent to the student. The student now uses this password along with the ID to request the type of result needed.

The system matches this password and generates the result, which is then sent back to the student. The password is generated once per result making it difficult to have just one permanent password for any transaction thus preventing multiple use.

The application queries the database and filters out the user's result appropriately after which it sends the examination result back to the user through the GSM terminal as shown in Figure 2. 


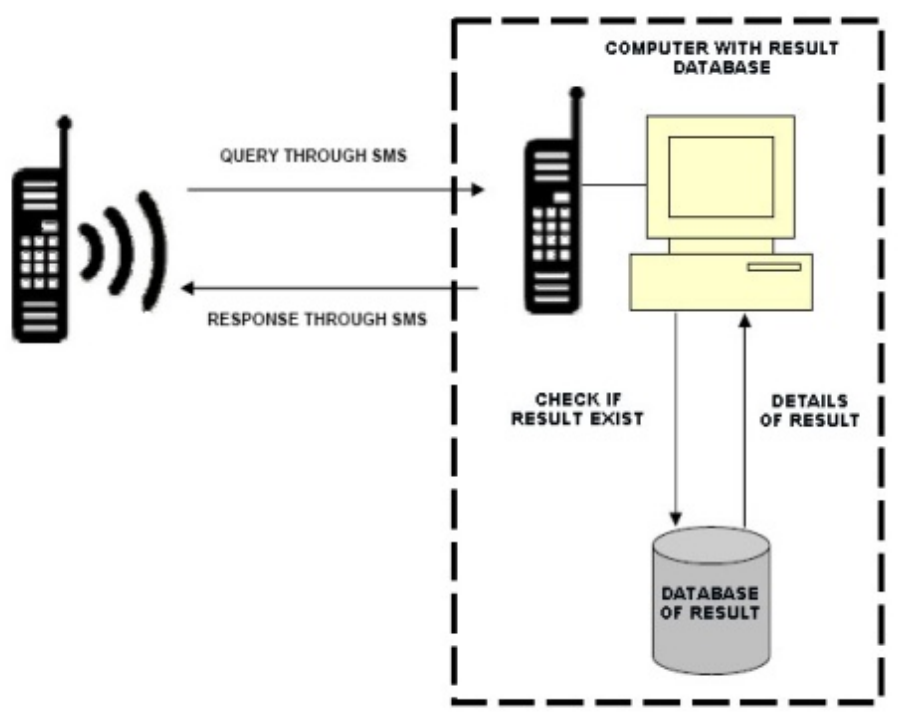

Figure 2: Functioning of a SMS Result Checking System

The flow of the systems starts when a student sends a SMS (in the prescribed format) to the given number. The system then uses the content of the SMS to process the student's request, after which the student's result is sent back to the student via the same number.

The features of this system are:

- It will receive SMS from all kind of phones from all networks.

- It functions $24 \mathrm{hrs}$ a day.

- Users are notified when they make an invalid request.

- It is relatively easy to customize.

- It uses passwords to provide security.

- It provides more flexibility, by allowing many result checking options.

\section{Security and Error Handling}

Like other means employed by institutions to disseminate examination results and other similar information, which includes website, email and IVRS, there exists the possibility of errors and security issues. While most universities make results available on their websites, students have to at least securely login to the website using their ID and a password. Apart from the possibility of a student knowing the ID and password of another student, the possibility of a guess can occur. Although, most websites offer a secure form of authentication like HTTPS, there is really no means of telling that the person that supplied the ID and password on the site is the actual student. Literature has proven that there have been more attacks on the web than SMS. All these imply that websites, though convenient to use, do not offer a totally secured solution for result checking.

Similarly, using emails or IVRS, there is no means of confirming that the person initiating a phone call on an IVRS system is the real student. And even in cases where the real student is the user of the system, a mistyped username and password would deny a student access to the site, and a student who mistakenly sends wrong information via email or an IVRS can obtain a false result, which could have consequences. An example of such event occurred in India, when a student, having probably sent the wrong details, got a false result and committed suicide (Wrong SMS leads to suicide, 2004). 
While each of these means has its own strength and weakness, it can be arguably said that no one is necessarily better than others. And as a matter of fact, several institutions use all these means together.

SMS on its own has several limitations, which include the limited number of characters allowed and the unavailability of features like printing, tables, images, etc. It, however, has almost the same security threat as the other means, considering the fact that same way a hacker can tap into a text message, the hacker can also hack into a school's website and pull results or manipulate the database, which is more disastrous.

The proposed system offers a reasonable level of security, which most similar systems do not provide. As discussed earlier, most SMS result checking systems either have student register their mobile phone number or have them send a text containing their identification number with no form of verification.

This system uses a password, with a default password assigned initially that they are expected to change on the school's website or by sending a SMS to the school. Specifying a password in the SMS ensures that if a cell phone gets stolen or get into the wrong hands, the student's result cannot be accessed unless the password is specified. This works in a similar way to the website, and neither of them has a way to prove that the request originates from the real user. Sharing or careless exposure of passwords will breach both systems; therefore education of users is paramount.

Error handling is an essential part of good system, as errors are bound to occur, especially in situations requiring user input. Users can misspell words, mismatch format, or even send the message to a wrong number. A feedback mechanism is therefore essential to inform users of their errors and provide suggestion of what went wrong. This feature is often missing in most SMS result checking systems; it is overlooked probably because the cost associated to sending SMS is low or even free. However, to ensure that users find the system friendly, error handling and notification is important. This system checks for possible errors in a received SMS and sends an error notification to the users. Possible errors are a wrong id and password combination, a wrong SMS format, etc.

\section{Implementation}

To use this system as an independent SMS service, the university must provide the necessary hardware and software requirement, which primarily are:

- A mobile phone with modem that supports communication with a computer, and a supported SIM card.

- A computer system to host the SMS application.

- A network connection to the database server.

- A cable or other connection means to connect the phone to the computer.

\section{System Verification}

As an example of the operation of this SMS result checking system, the mode of operation is illustrated using a particular $3^{\text {rd }}$ year student by the name "Bunmi David" with matriculation number " $05 / 0777$ ".

Bunmi David sends an SMS message with surname and ID. The system checks for Bunmi's name and generate password. Bunmi now re-send the password with the ID to request for result.

The SMS result checking, as shown in Figure 3, provides primarily two SMS formats. The first format requests for the detailed examination result, while the second format requests for the grade point average (GPA) only. As an advantage of this system over others, it allows students to check 
for results of past semesters, unlike most systems that only offer the current semester result. In this case, the student can specify which year and semester for which they want to access their grades. It is also possible to accept request for a particular course.
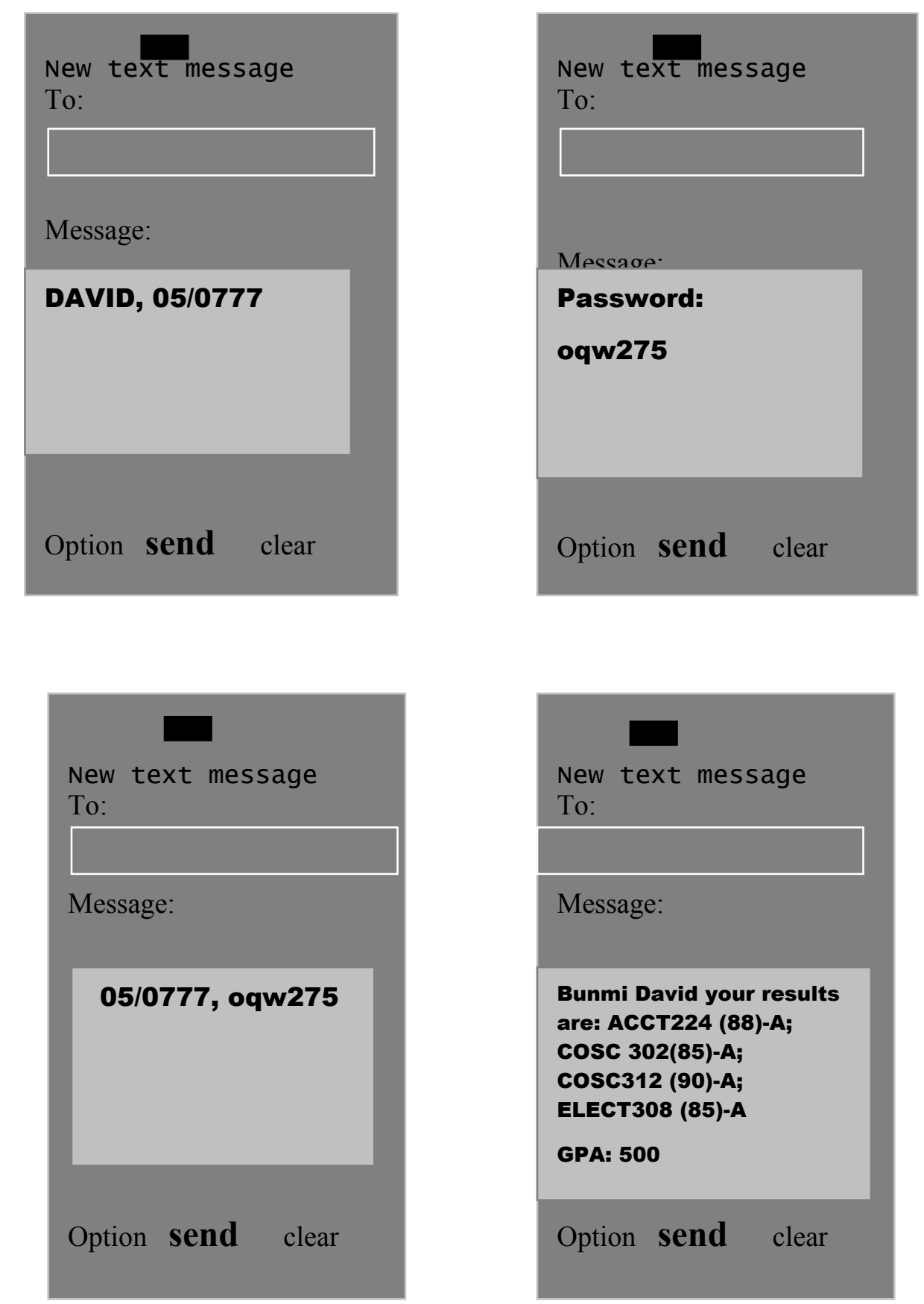

Figure 3: Example of SMS result checking system 


\section{Advantages of SMS Results Checking}

The advantages of SMS results are the same with that of any other SMS application.

- Convenience - Users get their results at their own convenience.

- Accessibility - Users can access their results from any location (as long as they are within a network service reception area).

- Portability - Results can be received and checked from any GSM phone. All GSM phones support SMS.

- Saves time - SMS is on the fly. It reduces the throughput of result checking as websites are sometimes unavailable due to congestion or server down time. SMS provides a faster means of sending and receiving such information.

- Cheaper-SMS is generally economical, and it sometimes is provided as a free service (at least for certain periods) by the service provider. Most providers also do not charge when users receive SMS.

- Less human resources required - Results are processed automatically

- Mobility - It is obvious that most mobile phone users have their phones with them everywhere they go; most people often leave their computer at home or work, but they will always have their phone on them.

\section{Limitations of SMS Results Checking}

The limitations of SMS results are the same with that SMS applications in general.

- Unlike paper results, which are stamped, it is difficult to certify SMS results. However, mobile operators provide numbers that bear the name of company or institution. This way the user can be sure that the message originated from the right source.

- As a rule, length of a SMS message is 160 characters. This is a limitation in SMS technology. Therefore the messages are abbreviated depending on the availability of the space.

- The SMS technology (like email) does not guarantee set transmission times or guaranteed delivery of the message; therefore some messages may be delayed, blocked, or lost in transmission.

- The cost of the message might be transferred to the sender (although toll-free lines can be acquired) and this is network dependent. It is also possible to reimburse such cost and to operate volume based tariffs.

- Service operators might not have coverage in some areas, and some locations may be have mobile-phone jammers therefore preventing users from getting service signals.

- Not all students will have a mobile phone. This is negligible considering the amount of mobile phone owners; at least one person in a family will own a phone.

\section{Conclusion and Further Work}

The SMS result checking system is a cost effective and widely available means of communication for most students. The use of this medium enhances easy access to their result. The previous paper (Adagunodo et al, 2009) described the use of the student's ID with a password for obtaining the result, while the improved system uses social interaction based on surname of the individual involved in the checking system to request for the scores in addition to the password system.

This system uses a 2-tier level verification system involving the student's surname. The SMS result checking system tries to approach examination result checking from the point of social interaction between the students to improve the security to a certain level. The former system needs the students to produce the ID and a password, while in the new system the password is self- 
generated. This system tries to develop an open system which is based on trust so that students can access their result without being limited to a particular phone number. The extent of response to messages will depend on the network in use; therefore, the guaranteed delivery of grades within the shortest period is networked based. Another limitation is that the phone may have a low battery at the point of delivery to a student whereby the phone is switched off on its own. Further work is still to be done on the security protocols and to really ascertain the delivery of the various messages to the recipients.

\title{
References
}

Adagunodo, E. R., Awodele, O., \& Ajayi, O. B. (2007). SMS Banking Services: A 21st Century Innovation in Banking Technology. Issues in Informing Science and Information Technology, 4, 101-112. Retrieved from http://proceedings.informingscience.org/InSITE2007/IISITv4p227-234Adag332.pdf

Adagunodo, E. R., Awodele, O., \& Idowu, O. (2009). SMS user interface result checking system. Issues in Informing Science and Information Technology, 6, 163-177. Retrieved from http://iisit.org/Vol6/IISITv6p101-112Adagunodo601.pdf

Desi, S. (2008). Check Orissa HSC exam results 2008/Board of Secondary Education. Retrieved from http://moviedrive.blogspot.com/2008/06/check-orissa-hsc-exam-results-2008.html

Ling, S. H (2005). SMS to get your exam results. Retrieved March 5, 2009 from http://www2.ntu.edu.sg/itzone/may2005/smsresult.html

Mavrakis, D. (2004). The Monaco Telematique mobile SMS whitepaper. Retrieved on December 1, 2008 from http://www.scribd.com/doc/916690/SMS-Whitepaper

Pramsane, S., \& Sanjaya, R. (2006). Mobile education services based on SMS and their architectture comparison. Retrieved on March 5, 2009 from http://www.ijcim.th.org/v14nSP1/pdf/p49.1-9-fin-61.pdf

Resource Shelf. (2006). Statistics: Use of text messaging (SMS) grows in the United States. Retrieved on March 5, 2009 from http://www.resourceshelf.com/2006/09/01/statistics- use-of-text-messaging-smsgrows-in-the-united-states/

Wrong SMS leads to suicide. (2004). Textually.org. Retrieved on March 5, 2009 from http://www.textually.org/textually/archives/2004/05/003929.htm

\section{Biographies}

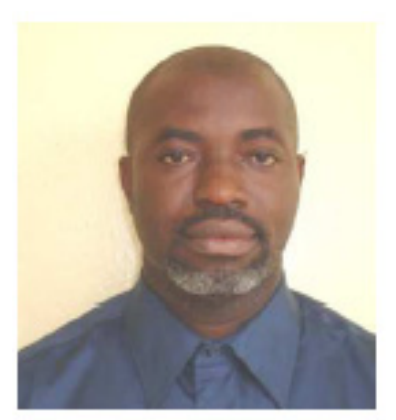

\begin{abstract}
Oludele Awodele has a Ph.D degree in Computer Science from the Universityof Agriculture, Abeokuta, Nigeria. He has several years of experience in teaching computer science courses at the university level. $\mathrm{He}$ is currently a lecturer in the department of Computer Science and Mathematics, Babcock University, Nigeria. He is a full member of the Nigeria Computer Society and the Computer Professional Registration Council of Nigeria. His areas of interest are Artificial Intelligence and Computer Architecture. He has published scientific articles in several journals of international repute.
\end{abstract}

Emmanuel Rotimi Adagunodo is a professor of computer science at the Obafemi Awolowo. University, Ile-Ife, Osun State, Nigeria. He is also the Deputy Director of Distance Learning. Center of the same University. He can be contacted at eadigun@oauife.edu.ng. 
Akinwale A. T. has a Ph.D degree in computer science, he is a senior lecturer and presently the HOD of the Department of Computer Science, University of Agriculture, Abeokuta Nigeria. His area of specialization is Knowledge Base system.

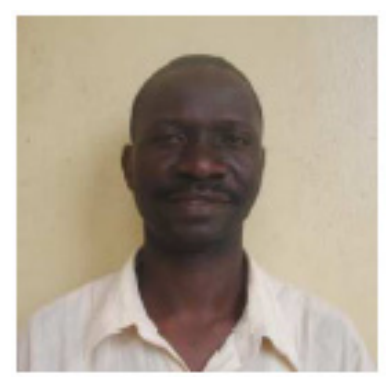

Sunday Idowu is a senior lecturer in the department of computer science \& mathematics, Babcock University, Ilishan-Remo, Ogun State, Nigeria. He holds a Masters degree in Software Engineering, and he is currently working on his Ph.D. in the University of Ibadan, Oyo State, Nigeria. His research areas are Software Engineering, Web Application Development and Security. He has published works in several journals of international repute. He can be contacted at saidowu06@hotmail.com

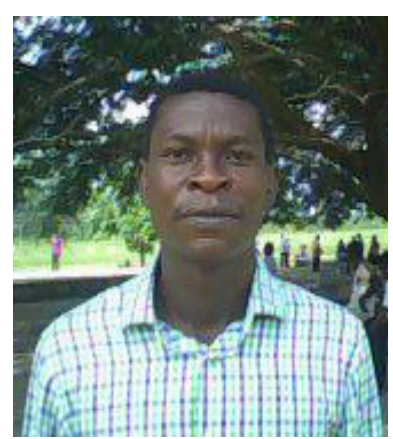

Agbaje M.O. is a lecturer in the Department of Computer Science, Babcock University, Illisan, Ogun State. He has a B.Sc. degree in Physics, from the University of Ilorin and M. Sc Computer Science, from the University of Agriculture, Abeokuta, Nigeria. His interests include: Information security, Artificial Intelligence and Embedded systems. 